REVIEW ARTICLE

\title{
Finding the sweet spot: glycosylation mediated regulation of intestinal inflammation
}

\author{
Jennifer C. Brazil ${ }^{1 凶}$ and Charles A. Parkos ${ }^{1 凶}$ \\ (c) The Author(s), under exclusive licence to Society for Mucosal Immunology 2021
}

Glycans are essential cellular components that facilitate a range of critical functions important for tissue development and mucosal homeostasis. Furthermore, specific alterations in glycosylation represent important diagnostic hallmarks of cancer that contribute to tumor cell dissociation, invasion, and metastasis. However, much less is known about how glycosylation contributes to the pathobiology of inflammatory mucosal diseases. Here we will review how epithelial and immune cell glycosylation regulates gut homeostasis and how inflammation-driven changes in glycosylation contribute to intestinal pathobiology.

Mucosal Immunology (2022) 15:211-222; https://doi.org/10.1038/s41385-021-00466-8

\section{INTRODUCTION}

Glycans are complex oligosaccharides that encode a vast range of distinct biological functions having remarkable implications in tissue homeostasis and disease. Decoration of glycoproteins with sugars regulates protein stability and generates the ligands for glycan-binding proteins (GBPs) including antibodies and lectins ${ }^{1}$. GBP-glycan binding interactions regulate key cellular processes including gene transcription, cell signaling, immune cell trafficking, pathogen-host interactions, and cellular differentiation ${ }^{2}$. Glycosylation regulates important processes in a wide variety of mucosal organs, including respiratory, gastrointestinal, and genitourinary tracts. This current review will specifically highlight how glycosylation contributes to homeostasis and inflammation in the gut.

\section{STRUCTURAL DIVERSITY AND COMPLEXITY OF N AND O-LINKED GLYCANS}

Mammalian oligosaccharide chains are generated through various combinations of ten individual monosaccharides: glucose (Glc), galactose (Gal), N-Acetylglucosamine (GlcNAc), $\mathrm{N}$-Acetylgalactosamine (GalNAc), glucuronic acid (GlcA), xylose (Xyl), mannose (Man), fucose (Fuc), Iduronic acid and sialic acid $(\mathrm{Sia})^{3}$. Interestingly while humans predominantly express the Sia variant $\mathrm{N}$-acetylneuraminic acid, the dominant Sia expressed by mice is $\mathrm{N}$-glycolylneuraminic acid ${ }^{4}$. Despite being generated from a somewhat limited set of monosaccharides, mammalian glycan chains display great structural diversity achieved by heterogenous topologies and a multitude of glycosidic linkages that connect individual sugar building blocks. Glycan diversity is further expanded by addition of monosaccharides in alpha or beta anomeric confirmations, multiple enzymatic site preferences as well as specific cell and environmental differences. Individual monosaccharides can be additionally modified by O-acetylation, O-sulfation and O-phosphorylation further adding to glycan structural heterogeneity. Importantly, this vast glycan complexity is regulated by $>700$ genes (including glycosyltransferases and sugar transporters) constituting $1-2 \%$ of the entire human genome ${ }^{5}$.

Mammalian glycan chains are most commonly attached to polypeptide structures through linkage to the amide Nitrogen of Asparagine ( $\mathrm{N}$-linked glycosylation) ${ }^{6}$ or through linkage to the hydroxyl group of Serine/Threonine (O-linked glycosylation) ${ }^{7}$. N glycan biosynthesis occurs in both the endoplasmic reticulum and Golgi apparatus, beginning with synthesis and transfer of a 14sugar precursor glycan $\left(\mathrm{Glc}_{3} \mathrm{Man}_{9} \mathrm{GlcNAc}_{2}\right)$ to a nascent polypeptide chain before removal of Glu and Man residues to form the $\mathrm{Man}_{3} \mathrm{GlcNAC}_{2}$ common N-glycan core structure (Fig. 1). This initial core $\mathrm{N}$-glycan structure can be further modified to form one of three main types of extended N-glycans: (1) oligomannosidic glycans in which only Man sugars are attached to the core; (2) complex glycans which have antennae formed from addition of GlcNAc to the common core structure; and (3) hybrid structures where only Man glycans are attached to the Mana1-6 arm of the core while one or two antennae are attached to the Mana1-3 arm (Fig. 1).

Unlike N-glycan biosynthesis, no oligosaccharide precursor is required for decoration of glycoproteins with O-linked glycans. The initiating event for O-linked glycosylation is addition of the monosaccharide GalNAc (from UDP-GalNAc) to Ser/Thr residues on an acceptor glycoprotein. The majority of O-linked glycans fall into four main categories referred to as Core 1-4 structures (Fig. 1). The initial O-glycan structure linked to Ser or Thr, referred to as Thomsen-nouveau (Tn) antigen (GalNAca-O-Ser/ Thr), is subsequently extended by additional monosaccharides transferred to growing glycan chains by specific glycosyltransferases. A ubiquitously expressed glycosyltransferase (Core 1 ß1-3-galactosyltransferase, C1GalT1) adds Gal to Tn structures forming Core 1 type O-glycans (Galß1-3GalNAC) ${ }^{8,9}$. An additional glycosyltransferase, Core $3 \quad \beta 1-3-N-$ Acetylglucosaminyltransferase (C3GnT), adds GlcNAc to $\mathrm{Tn}$ structures to form core 3 derived O-glycans ${ }^{10,11}$ (Fig. 1). Core

1Department of Pathology, University of Michigan, Ann Arbor, MI 48109, USA. ${ }^{凶}$ email: brazilj@umich.edu; cparkos@umich.edu 


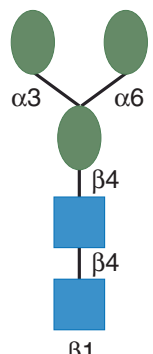

Common $\mathrm{N}$ glycan core b

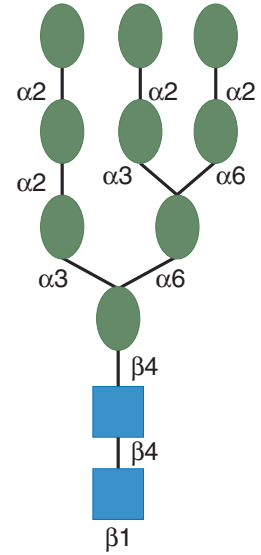

High mannose

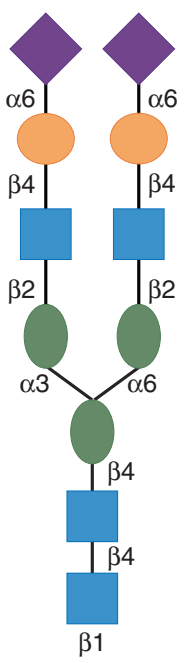

Complex

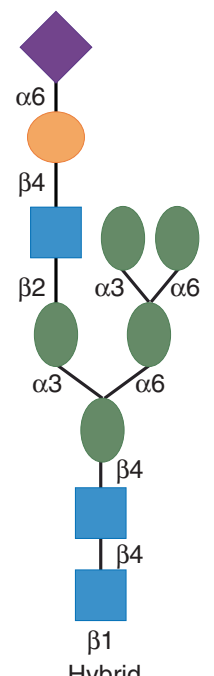

Hybrid
Extended N glycans c

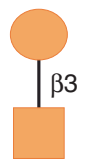

$\alpha 1$

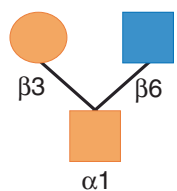

Core 2

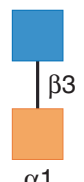

Core 3

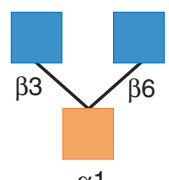

$\alpha 1$ d

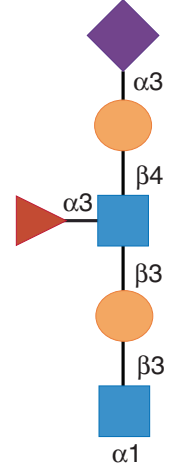

Extended core 1

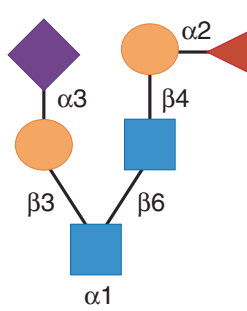

Extended core 2

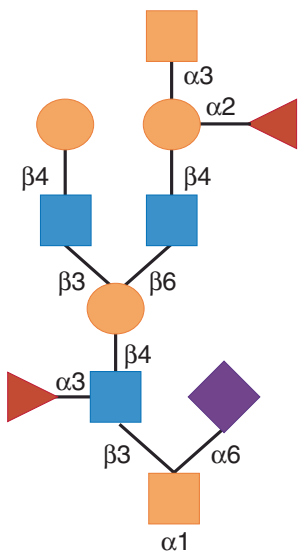

Extended core 3

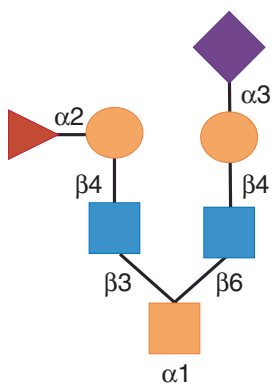

Extended core 4
Extended $\mathrm{O}$ glycans

O Glycan cores

Key:

Man

GlcNAc

Gal

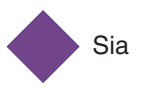

Fuc

GalNAc

Fig. 1 Common core and extended $\mathbf{N}$ and $\mathbf{0 - g l y c a n}$ structures. a Depiction of common $\mathbf{N}$-glycan core structure. $\mathbf{b}$ Three main types of extended N-glycans (High Mannose, Complex, and Hybrid) share a common core structure including the first two GlcNAc residues and the first three Man residues. c Depiction of Core 1-4 O-glycan structures. d Representative examples of complex O glycans with extended core 1, 2,3 or 4 structures.

1 and Core 3 O-glycans can be modified by the addition of a branching GICNAc at the C6 hydroxyl group to form Core 2 or Core 4 structures, respectively ${ }^{12,13}$ (Fig. 1). Individual Core O-glycan structures must be correctly assembled by the relevant glycosyltransferases to facilitate subsequent O-glycan chain extension and generation of finalized glycan structures that are required for proper glycoprotein function. As glycoproteins traverse the Golgi apparatus, $\mathrm{O}$ glycan core structures are modified by sequential addition of Gal, GlcNAc, GalNAc, Fuc, Sia, and sulfate to form more complex extended glycan structures of varying lengths. Importantly, while Core 1 and Core 2 structures are expressed in most tissues, Core 3 and Core 4 O-glycans are exclusively expressed in the intestinal epithelium ${ }^{14-16}$
INFLAMMATION-INDUCED ALTERATIONS IN GLYCOSYLATION IN THE INTESTINAL EPITHELIUM ARE LINKED TO PATHOBIOLOGY OF MUCOSAL DISEASE

The intestinal epithelium expresses a plethora of $\mathrm{N}$ and O-linked glycans that control key functions important for barrier function and protection against foreign toxins and pathogens. As such intestinal epithelial cell glycans play a key role in maintaining mucosal homeostasis despite being constantly challenged by diverse foreign stimuli. Mucin type O-glycans are the major class of glycans expressed by intestinal epithelial cells with differential core O-glycan expression observed throughout the human digestive tract (Fig. 2). Core-1 and Core-2 O-glycans are expressed by epithelial cells in the stomach and duodenum ${ }^{17}$. In contrast, Core-3 O-glycans are expressed by epithelial cells in the jejunum 


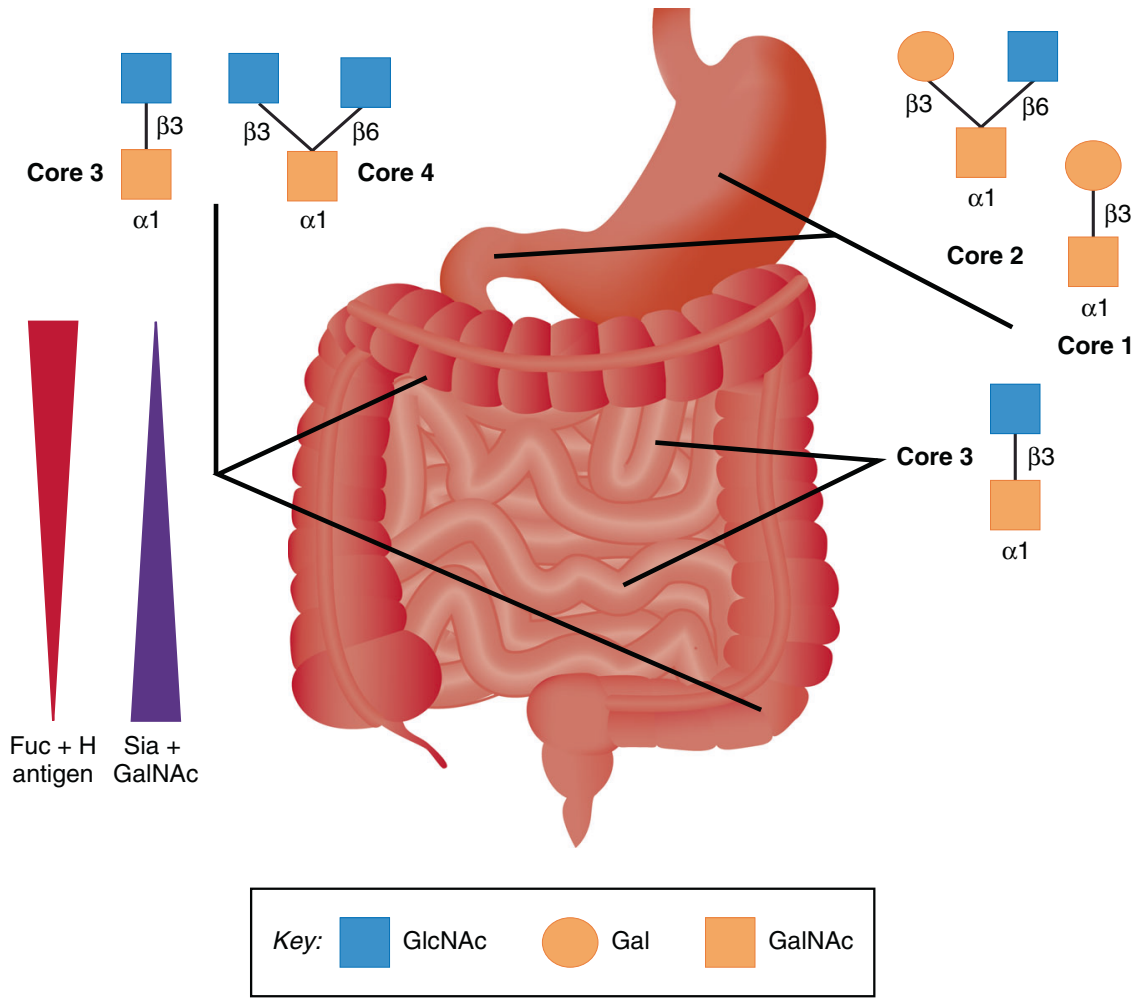

Fig. 2 Differential glycan expression throughout the gastrointestinal tract. Cartoon depicting expression of Core O-glycans and terminal glycans in the human gastrointestinal tract. Core-1 and Core-2 O-glycans are expressed by epithelial cells in the stomach and duodenum. In contrast Core-3 O-glycans are expressed by epithelial cells in the jejunum and ileum while colonic epithelial cells mostly express Core-3 and Core-4 glycans. Figure also depicts the increased expression of terminal Sia and GalNAc and decreased expression of terminal Fuc observed as you move from the stomach to the rectum.

and ileum while colonic epithelial cells mostly express Core-3 and Core-4 $_{\text {glycans }}{ }^{18-20}$ (Fig. 2). Interestingly, terminal glycosylation in humans is also regulated regionally by host and environmental factors in the intestine from the stomach to the rectum with increased levels of terminal Sia and GalNAc and decreased levels of glycans terminating with Fuc in the distal colon ${ }^{17,21}$. In contrast to human gut glycosylation patterns, the murine intestinal glycome consists primarily of Core-2 based O-glycans ${ }^{22,23}$. Furthermore, while glycosylated structures in murine ileum, jejunum, and duodenum are dominated by sialylated and sulfated Core-2 O-glycans, mouse colon predominantly displays highly charged fucosylated glycans ${ }^{22,23}$. Despite some differences in glycan localization, mice and humans express many of the same terminal gut glycan epitopes including Sia, sulfate, and Fuc, making murine models suitable for studying many aspects pertaining to regulation of intestinal epithelial glycosylation.

Epithelial glycans in the intestine serve as ligands that induce host immune signaling and act as nutrients that regulate gut microbial composition ${ }^{24-30}$. Furthermore, recent studies have highlighted that human and murine small intestinal Paneth cells express specific N-Acetyllactosamine (Galß1-4GlcNAc, LacNAc) glycans that control growth and differentiation of adjacent stem cells, a critical event for ongoing intestinal epithelial selfrenewal $^{31,32}$. Therefore, intestinal epithelial glycans play an essential role in integrating host, microbial, and environmental cues to maintain mucosal homeostasis.

In addition to regulating mucosal homeostasis, altered intestinal epithelial glycan expression is implicated in the complex etiology of several inflammatory diseases of the gut including both forms of inflammatory bowel disease (IBD), ulcerative colitis (UC), and Crohn's disease, with aberrant glycan expression directly contributing to disease pathophysiology ${ }^{33,34}$. Altered cell surface glycosylation can be triggered by disrupted intracellular glycan biosynthesis, changes in exposure of surface glycans to glycan modifying enzymes (glycosidases) or changes in expression of underlying substrate glycoproteins. Interestingly, of the hundreds of genes involved in glycan biosynthesis, at least 20 have been

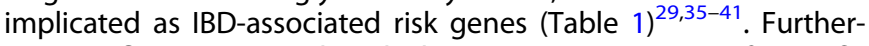
more, inflammatory induced changes in expression of specific epithelial glycoproteins as well as altered activity of glycan modifying enzymes such as sialidases has been identified in the intestinal mucosa of people with UC and Crohn's disease. ${ }^{33,34,42-44}$.

One of the most common alterations in epithelial glycosylation observed under conditions of chronic intestinal inflammation is upregulated expression of truncated or immature surface glycans (Fig. 3). For example, increased epithelial expression of the oncofetal Thomsen-Friedenreich (TF) disaccharide Galß1-3GalNAc is observed in the intestinal mucosa of individuals with UC and in Cotton-top tamarins with chronic colitis ${ }^{45,46}$. The TF antigen is recognized by the plant lectin peanut agglutinin $(P N A)^{47}$, and increased binding of PNA is a common feature in the inflamed mucosa of individuals with $\mathrm{IBD}^{48-50}$. Interestingly, increased TF antigen expression is also a feature of epithelial hyperplasia observed in colorectal tumors ${ }^{51}$. Given the association between chronic colitis and cancer, it can be speculated that immature epithelial glycans are a common feature driving IBD pathogenesis and gastrointestinal malignancy. Importantly, studies of unaffected monozygotic twins of individuals with IBD have revealed elevated TF antigen expression in intestinal epithelial crypts that is associated with increased NF-KB activation ${ }^{52}$. These findings suggest that changes in epithelial glycosylation precede disease initiation and that induction of TF antigen expression might predict chronic inflammatory responses in the gut ${ }^{52}$. While the role of epithelial Gal $\beta 1-3-G a I N A c$ in IBD pathogenesis remains incompletely understood, it has been proposed that increased intestinal epithelial TF expression facilitates binding interactions 
Table 1. Inflammatory bowel disease risk associated genes involved with glycosylation.

\begin{tabular}{|c|c|}
\hline \multicolumn{2}{|c|}{ Ulcerative colitis only } \\
\hline HNF4A & $\begin{array}{l}\text { Transcription Factor that regulates Plasma protein } \\
\text { fucosylation } 188,189\end{array}$ \\
\hline MANBA & $\beta-$ Mannosidase ${ }^{190,191}$ \\
\hline MAN2A1 & $\alpha-$ Mannosidase $^{38}$ \\
\hline SLC9A3 & $\begin{array}{l}\text { Solute carrier family } 9 \text { (Sodium/Hydrogen Exchanger), } \\
\text { target of PHA lectin }{ }^{192,} 193\end{array}$ \\
\hline \multicolumn{2}{|c|}{ Crohn's disease only } \\
\hline IL6ST & Cytokine that regulates IgG glycosylation ${ }^{39,41}$ \\
\hline Fut2 & $\alpha 1,2$ Fucosyltransferase ${ }^{36}$ \\
\hline LGALS9 & Gal binding lectin (Galectin-9) ${ }^{45}$ \\
\hline \multicolumn{2}{|c|}{ IBD (both ulcerative colitis and Crohn's disease) } \\
\hline IL10 & $\begin{array}{l}\text { Cytokine that regulates intestinal epithelial } \\
\text { fucosylation }^{28,194,195}\end{array}$ \\
\hline GALC & $\begin{array}{l}\text { Galactosylceramidase that hydrolyzes galactose ester } \\
\text { bonds }^{196}\end{array}$ \\
\hline TMEM258 & $\begin{array}{l}\text { Component of the oligosaccharyltransferase } \\
\text { transferase complex }{ }^{197}\end{array}$ \\
\hline C1GALT1C1 & $\begin{array}{l}\text { X linked chaperone protein involved in core } 1 / \text { core } 2 \\
\text { O-glycan biosynthesis } 35\end{array}$ \\
\hline SELE & Sia binding lectin (E-Selectin) ${ }^{198}$ \\
\hline SELL & Sia binding lectin (L-Selectin) ${ }^{198}$ \\
\hline SELP & Sia binding lectin (P-Selectin) ${ }^{198}$ \\
\hline RORC & $\begin{array}{l}\text { Transcription Factor that regulates immune T-cell } \\
\text { glycosylation } 199\end{array}$ \\
\hline IKZF1 & $\begin{array}{l}\text { Transcription Factor that regulates IgG N } \\
\text { glycosylation } 39,65,199\end{array}$ \\
\hline MGAT3 & $\begin{array}{l}\text { Transcription Factor that regulates IgG N } \\
\text { glycosylation }^{39}\end{array}$ \\
\hline $\mathrm{BACH} 2$ & $\begin{array}{l}\text { B cell specific transcription factor that regulates lgG } \\
\text { glycosylation } 39,65,200\end{array}$ \\
\hline IL23R & Highly glycosylated cytokine receptor ${ }^{199,} 201$ \\
\hline IL2RA & Cytokine implicated in N-glycan branching 202,203 \\
\hline
\end{tabular}

between Galß1-3-GalNAc and mitogenic lectins of dietary and microbial origin that drive epithelial hyper-proliferation responses ${ }^{53,54}$. Intriguingly, it has been demonstrated that PNA coated betamethasone-containing nanoparticles specifically target inflamed intestinal epithelium in vivo and reduce pathological inflammation in both TNBS and oxazolone mediated models of murine colitis ${ }^{55}$. Similarly, the Fusobacterium nucleatum lectin Fap2 binds to Galß1-3-GalNAc and facilitates bacterial targeting to inflamed TF-expressing intestinal epithelium in vivo ${ }^{25}$. Importantly, growing evidence suggests that $F$. nucleatum is a pathogenic bacteria, enriched in the mucosa of people with UC, that triggers autophagic epithelial cell death pathways to exacerbate intestinal inflammation ${ }^{56}$.

Together with changes in Gal containing glycans, studies have highlighted dynamic changes in epithelial fucosylation during intestinal inflammation ${ }^{57,58}$. Moreover, genetic deficiencies in fucosylation have been shown to inhibit intestinal Notch signaling and trigger spontaneous colitis and adenocarcinoma ${ }^{59}$. Fucosyltransferases add Fuc to growing glycan chains in a1-2, a1-3, a1-4 or a1-6 conformations, with a1-2 being the dominant Fuc linkage found in the intestinal epithelium of humans and mice ${ }^{60-64}$. Genome-wide association studies (GWAS) have identified strong associations between IBD susceptibility and polymorphisms in intestinal a1-2 fucosyltransferases (FUT1 and FUT2) ${ }^{36,65-68}$. ABO blood group antigens are formed by modification of terminal Gal residues by addition of a1,2 Fuc to form Fuca1-2Gal (H antigen). FUT2 encodes the $\mathrm{H}$ antigen in intestinal epithelial cells lining the gastrointestinal tract while FUT1 is necessary for synthesis of these structures on erythrocytes ${ }^{69}$. Epithelial expressed ABO-related antigens strongly influence gut microbial composition by functioning as binding epitopes for certain intestinal microbes including Helicobacter pylori and Norovirus and acting as a Carbon source for bacterial species such as Escherichia coli ${ }^{70,71}$. Recent studies have demonstrated that during homeostasis bacterial interactions with innate lymphoid cells triggers production of IL22 which binds to IL-22R and induces epithelial expression of a1-2 Fuc in mice ${ }^{29,72}$. Increased epithelial a1-2 Fuc expression has been shown to reduce colonization by opportunistic gut pathogens including Enterococcus faecalis while promoting growth of commensal microbes such as Porphyromonadaceae, Ruminococcaceae, and Bacteroides species ${ }^{29,72}$. Importantly, loss of function mutations in FUT2 have been shown to directly contribute to colitis associated microbial dysbiosis in individuals with Crohn's disease ${ }^{37}$. While loss of function mutations in FUT2 represent IBDassociated genetic risk factors ${ }^{36,37}$, elevated intestinal epithelial FUT2 expression has also been reported in the inflamed mucosa of people with Crohn's disease ${ }^{73}$. Others have demonstrated that IL10 producing lamina propria $\mathrm{CD}^{+} \mathrm{T}$ cells suppress murine intestinal epithelial FUT2 expression ${ }^{28}$, suggesting complex regulation of intestinal fucosylation is required to maintain mucosal homeostasis in the gut.

In conjunction with the role of epithelial expressed a1-2 Fuc, oral delivery of exogenous Fuc has been shown to alleviate chronic dextran sodium sulfate (DSS) induced colitis in mice by decreasing inflammation-induced intestinal bile acid accumulation and shifting overall bile acid profiles to favor colonization by beneficial commensal gut microbes ${ }^{74,75}$. Supplementation with Fuc has also been shown to ameliorate acute DSS colitis by limiting macrophage $M 1$ polarization and inhibiting NLRP3 inflammasome and NF-kB activation ${ }^{76}$. These findings serve to highlight the importance of epithelial fucosylation for maintenance of microbial eubiosis in the gut as well as the complexity of a1-2 Fuc contributions to regulation of intestinal epithelial function by components of the innate and adaptive immune systems.

Consistent with reports showing altered intestinal fucosylation, significant changes in epithelial sialylation are observed within intestinal mucosa of people with UC and Crohn's disease. Increased expression of short O-linked oncofetal glycans such as the sialyl Tn antigen (Siaa2-6GalNAc) occur in inflamed mucosa from these individuals ${ }^{77-79}$. CD44 type I transmembrane glycoproteins represent an important family of heavily sialylated adhesion molecules expressed by intestinal epithelial cells. Immunohistochemical studies of human intestinal mucosa have shown that expression of the CD44v6 variant is upregulated during colitis and in intestinal tumors of epithelial origin ${ }^{42,43,80,81}$. Importantly, increased CD44v6 expression in IBD correlates with increased inflammatory infiltrates and with increased mucosal tissue damage ${ }^{42}$. More recent immunohistological and liquid chromatography mass spectrometric glycomics analyses have demonstrated upregulated expression of sialylated Lewis family O-glycans on epithelial CD44v6 and on Muc2 in inflamed human and murine colonic mucosa ${ }^{33,34,82-85}$. Furthermore, recent functional studies have shown that antibody targeting of specific sialyl-Lewis glycans on epithelial CD44v6 blocks human neutrophil transepithelial migration (TEpM), protects against DSS-induced colitis in vivo and promotes epithelial wound closure following colonic injury ${ }^{33,34,86}$. These studies highlight how inflammation induced sialylated O-linked glycan epitopes may represent potential therapeutic targets for limiting excessive PMN infiltration, driving re-epithelization processes, and promoting restoration of mucosal homeostasis in the inflamed gut. 

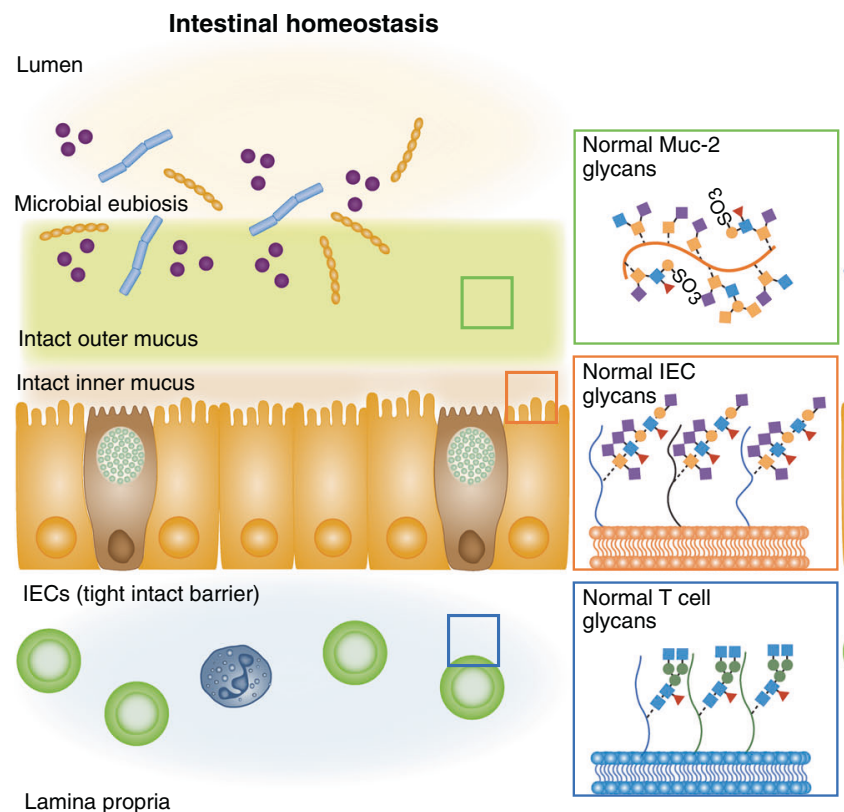

Lumen
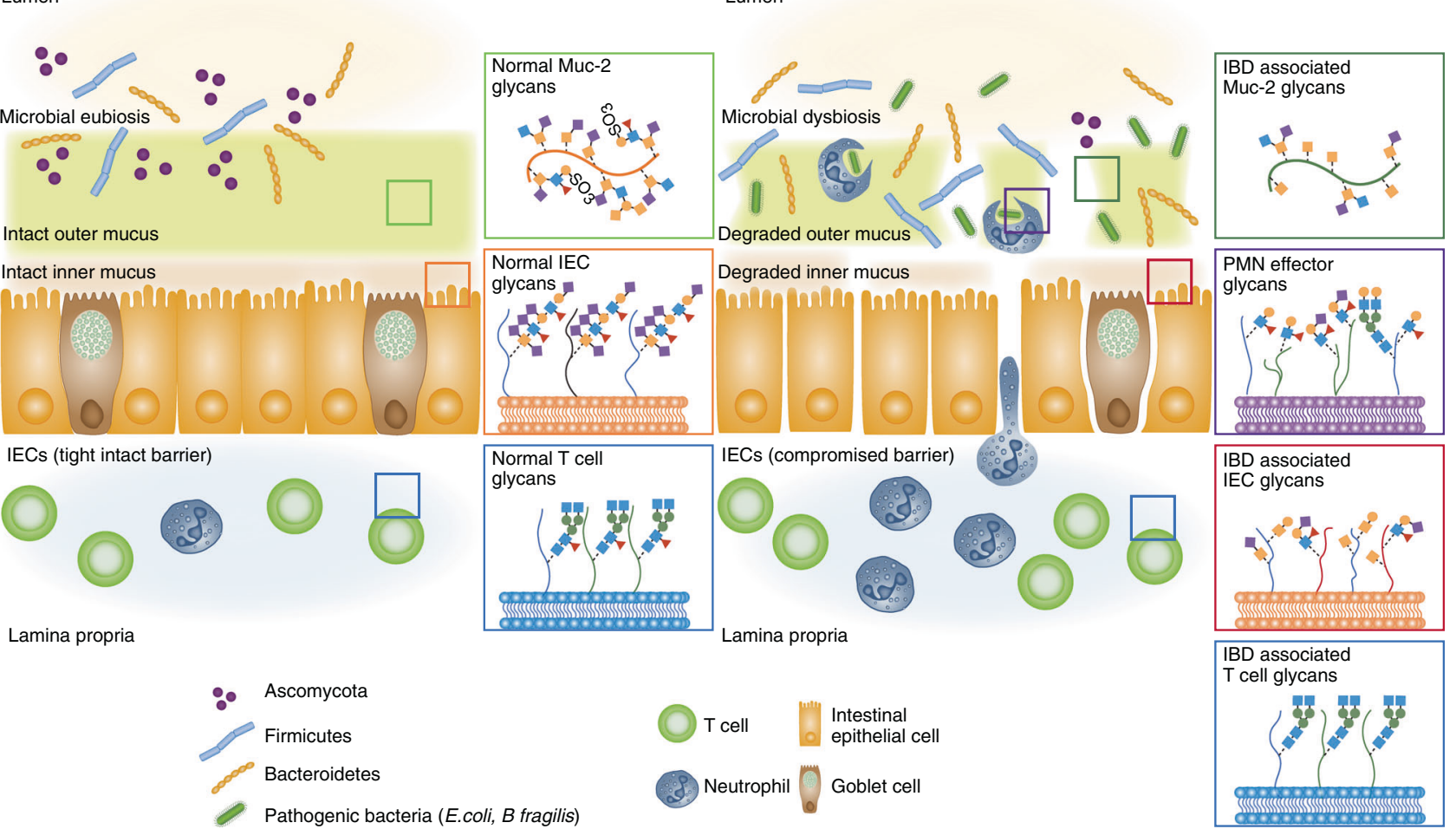

Fig. 3 Model showing changes in mucosal glycosylation during intestinal inflammation. Left panel shows intestinal homeostasis with intact, mucus layers, microbial eubiosis, and expression of mature surface glycans. Right panel depicts intestinal inflammation with degraded mucus layers, overgrowth of pathogenic bacteria, increased PMN trafficking to the lumen and altered surface expression of glycan structures on epithelial cells, immune cells, and intestinal mucins ${ }^{15,34,64,77-80,90,91,95-100 . ~}$

\section{ABERRANT GLYCOSYLATION OF INTESTINAL MUCINS IS OBSERVED IN IBD}

The physical barrier provided by absorptive enterocytes is augmented by hyper O-glycosylated mucin proteins secreted by goblet cells (GCs) to provide an additional layer of protection against intestinal pathogens (Fig. 3). A recent single cell RNA-seq study highlighted a sub-population of intestinal GCs that upregulate stress response genes in the mucosa of individuals with UC suggesting dynamic differentiation of GCs during intestinal inflammation ${ }^{87}$. Furthermore, another RNA-seq analysis in mCherry-Muc2 transgenic mice identified a sub-population of highly differentiated colonic GCs that localize to the surface epithelium between intestinal crypts (inter-crypt GCs, icGCs). Functionally icGCs were shown to have a distinctive transcription profile producing a more permeable mucus that fills spatial regions between mucus plumes released from standard intestinal crypt GCs. Importantly, reduced numbers of icGCs along with disorganized mucus structure was observed during DSS colitis in mice and in the intestinal mucosa of individuals with UC highlighting important regulation of GC maturation and transcription during intestinal inflammation ${ }^{88}$.

In the small intestine of humans and mice, Muc2 is the most abundant mucin and forms a single loose layer of mucus that is penetrable by certain bacteria thus serving as a habitat for commensal gut microbes ${ }^{89}$. Structurally Muc2 resembles a bottle brush with a protein core at its center and glycans (which account for $80 \%$ of molecular mass) acting as bristles. In the colon, where there are as many as $10^{12}$ bacteria per gram of stool, Muc2 forms an outer non-attached mucus layer inhabited by microbes and a sterile inner mucus layer that is adherent to the intestinal epithelium ${ }^{90,91}$. The sterile inner mucus layer of the distal colon is renewed by GC's every hour and is gradually converted into the outer more permeable mucus layer via host-controlled proteolytic cleavage of cysteine rich regions of Muc2 ${ }^{92}$. Such cleavage does not disrupt Muc2 polymerization but rather allows the mucus to expand to approximately four times its volume that makes a more soluble habitat to support intestinal microbes ${ }^{92}$. Bacteria resident in the loose mucus gel of the intestine use glycan-degrading enzymes to sequentially release monosaccharides from mucin glycans until the protein core is exposed and degraded by proteolytic enzymes ${ }^{92}$. Monosaccharides released in this fashion can be metabolized by bacteria into short fatty acids such as butyrate that are utilized as an energy source by intestinal enterocytes, highlighting that sugar-derived glycan metabolites facilitate a symbiosis between commensal microbes and intestinal epithelial cells ${ }^{93,94}$. The importance of intestinal microbiota for mucus glycosylation is further highlighted by work comparing Muc2 glycosylation in germ-free mice and mice raised under standard housing conditions. Increased glycosyltransferase expression along with more extended glycan structures and higher levels of fucosylation on intestinal Muc2 has been reported in conventionally raised mice ${ }^{22}$ suggesting that commensal microbes play an important role in the glycosylation of intestinal Muc2. In addition to providing a food source for intestinal microbes and minimizing bacterial-epithelial contact, murine intestinal Muc2 O-glycans have been reported to regulate mucosal homeostasis by forming an immune complex with Galectin-3, Dectin-1, and FcyRIIB that inhibits pro-inflammatory cytokine production by dendritic cells ${ }^{26}$.

Intestinal mucin glycans are often modified by sulfation and, mice lacking intestinal sulfate transporters (or sulfate transferases) display increased leukocyte trafficking to the gut, enhanced intestinal permeability, and increased susceptibility to DSS induced colitis ${ }^{95,96}$. Moreover, histochemical analyses have demonstrated decreased sulfation of Muc2 O-glycans in colonic biopsy specimens isolated from individuals with active UC ${ }^{97-100}$. 
As such, decreased sulfation of Muc2 glycans, as observed in UC, is likely to be important for disease pathogenesis although specific mechanisms for this have yet to be fully elucidated. Interestingly, despite having some common alterations in intestinal glycosylation, South Asians who develop IBD differ from their European counterparts in that they do not have reduced levels of Muc2 glycan sulfation ${ }^{101}$. Importantly, South Asians have lower overall incidence rates for IBD, and colitis-associated cancer compared to North Americans and Europeans ${ }^{101,102}$. Therefore, it can be speculated that lower rates of IBD and colitis-associated cancer observed in certain populations could be related to specific regional differences in intestinal glycan sulfation.

Increased expression of truncated or shortened O-glycans such as the cancer-associated Tn antigen on Muc2 has also been reported in patients with active $U C^{15,79,82}$. Furthermore, intestinal Muc2 glycosylation was found to be similar across normal controls and UC patients in remission suggesting that inflammatory mechanisms regulate post translational glycosylation of intestinal Muc2 and, that IBD-associated changes in glycosylation are reversible over time ${ }^{82}$. Another consequence of expression of truncated or immature O-linked intestinal glycans is the loss of epithelial expressed sulfo-Lewis glycans that represent ligands for specific immune cell expressed Sia binding immunoglobulin-like lectins (Siglecs) including Siglec $9^{82,83,103}$. While the importance of Siglec 9 signaling during intestinal inflammation has not been studied to date, previous studies have demonstrated that engagement of Siglec-9 triggers programmed cell death responses in human neutrophils (PMN) ${ }^{104}$. Other studies have reported regulation of PMN trafficking into inflamed lung tissues by the murine analog of Siglec 9 (Siglec E) ${ }^{105,106}$. Therefore, it is likely that loss of inhibitory Siglec 9 signaling in the intestine might promote hyperactivation of PMN which would contribute to the chronic mucosal inflammatory responses observed in IBD.

The importance of O-glycans in maintenance of intestinal homeostasis is exemplified in mice lacking Core-3 derived glycosyltransferases. These mice display reduced levels of total intestinal O-glycans and are more susceptible to DSS-induced colitis $^{14}$. Similarly, mice lacking intestinal Core-1 mucin-type O-glycans develop spontaneous colitis that is triggered by microbiota and epithelial-derived caspase- 1 dependent mucosal inflammasome activation ${ }^{107}$. Indeed, mice with intestinal epithelial-specific deficiency of Core-1 derived O-glycans (C1GALT1 IEC knockout mice) have been shown to develop spontaneous distal colitis with massive neutrophilic infiltrates, crypt abscesses and histopathology that closely resembles $\mathrm{UC}^{15}$. Successful functioning of C1GALT1 requires the $X$ linked chaperone protein Cosmc (C1GALT1C1) $)^{15,16,108}$. GWAS analyses have shown that loss of function mutations in Cosmc represent IBD risk-associated genes ${ }^{35,109}$, suggesting that lack of intestinal C1GALT1 activity contributes to the aberrant intestinal $O$ glycosylation observed in individuals with UC. While the role epithelial glycosylation plays in driving intestinal inflammation is an emerging area of interest, studies to date highlight that altered intestinal O-glycosylation results in a defective inner colonic mucus layer, increased contact between epithelial cells and bacteria, and triggering of pro-inflammatory immune responses that likely contribute to IBD pathogenesis (Fig. 3).

\section{IGG GLYCOSYLATION AND EFFECTOR FUNCTION ALTERATIONS IN IBD}

Together with inflammation-induced changes in intestinal epithelial glycan composition altered glycosylation and distribution of serum IgG occurs in IBD. Glycosylation of IgG modulates binding affinity to FcyRs instructing either pro or anti-inflammatory responses ${ }^{110,111}$. Interestingly, 5 of the 16 genes that regulate IgG glycosylation contain SNPs linked to increased risk for IBD identified by GWAS ${ }^{39}$. Reduced levels of serum IgG sialylation is observed in both UC and Crohn's disease ${ }^{112-114}$ as well as altered fucosylation, with increased levels detected in Crohn's disease and lower levels in people with UC $\mathrm{U}^{112,115}$. While the functional role of IgG fucosylation in regulating intestinal inflammation is not understood, afucosylated IgG has been shown to enhance proinflammatory cytokine production by monocytes in the lungs of individuals with severe COVID-19 ${ }^{116}$, suggesting that altered IgG fucosylation could also contribute to IBD pathobiology. Importantly, the prevalence of agalactosylated IgG increases in Crohn's disease patients where it correlates with levels of $C$ reactive protein suggesting that changes in IgG galactosylation directly contribute to IBD disease severity ${ }^{117-119}$. One of the important consequences of $\mathrm{lgG}$ galactosylation is exposure of underlying GlcNAc glycans that have been shown to bind to mannan-binding lectin and induce inflammation via activation of the lectin complement pathway in rheumatoid arthritis ${ }^{120}$. Given that mannan-binding lectin expression is upregulated during intestinal inflammation ${ }^{12} \mathrm{~T}$, it can be speculated that aberrant lgG galactosylation also contributes to IBD pathobiology through activation of inflammatory signaling pathways in the gut. Overall, a clear functional role for IgG glycosylation in IBD pathophysiology has yet to be determined. However given that current strategies for differential diagnosis and monitoring of IBD can lack sensitivity and are often invasive ${ }^{122,123}$, measurement of serum $\operatorname{lgG}$ glycosylation could potentially represent an attractive and minimally invasive option for IBD patient stratification ${ }^{114,115}$.

Along with changes in IgG glycosylation, serological studies have shown that exposure of host cells to surface glycans expressed by intestinal bacteria triggers the production of carbohydrate-specific antibodies. Indeed, people with IBD commonly have elevated titers of such glycan reactive anti-microbial antibodies ${ }^{124-129}$. In IBD, there is decreased microbial diversity and increased colonization of potentially inflammatory bacterial species including adherent-invasive $E$. coli $i^{130-132}$. Since certain pathogenic bacteria mimic host glycans to evade immune recognition ${ }^{133,134}$, antibodies against bacterial glycans have the potential to cross react with intestinal epithelial glycoepitopes and contribute to the chronic inflammatory responses observed during active flares of IBD.

\section{NEUTROPHIL GLYCOSYLATION AND INTESTINAL INFLAMMATION}

In addition to intestinal epithelial cell dysfunction and altered IgG glycosylation, dysregulated neutrophil (PMN) infiltration and activation are closely linked to pathobiology in $\mathrm{IBD}^{135-139}$. It is well appreciated that in the gut, uncontrolled influx of PMNs across intestinal epithelial barriers coupled with indiscriminate release of toxic reactive oxygen metabolites and tissue degrading proteases results in extensive mucosal and/or transmural injury including edema, loss of GCs, decreased mucus production, crypt injury with erosions, ulceration and, crypt abscess formation ${ }^{135-137,140,141}$. Importantly, critical binding interactions facilitating PMN recruitment out of the microcirculation are regulated by cellular glycosylation $^{142,143}$. In particular endothelial E and P-selectins engage sialyl-Lewis $X$ [Sia-a2-3Galß1-4(Fuca1-3)GlcNAc-R, sLe ${ }^{x}$ containing $\mathrm{N}$ - and $\mathrm{O}$ - glycans on PMN glycoproteins including CD44, E-selectin ligand 1 and P-selectin glycoprotein ligand 1 to facilitate PMN tethering and rolling (reviewed elsewhere ${ }^{143}$ ). Firm adhesion of PMN to endothelial cells is further mediated by interactions between PMN integrins and oligomannosidic $\mathrm{N}$-glycans displayed on endothelial ICAM-1 ${ }^{144-149}$.

While mechanisms that drive PMN extravasation are relatively well described, less is known about how PMN cross the epithelial barriers that line mucosal organs such as the intestine. However, more recent studies have highlighted an important role for glycan-mediated binding events in regulating PMN TEpM. It has been reported that $s L e^{x}$ glycans on PMN CD11b/CD18 mediate 
binding interactions with intestinal epithelial expressed ICAM-1 and, that antibody ligation of $s L e^{x}$ induces human PMN aggregation and degranulation responses while reducing levels of PMN TEPM ${ }^{150}$. In addition to sialylated glycans regulating PMNintestinal epithelial interactions, fucoidin (a homopolymer of sulfated L-Fuc) reduces levels of PMN TEPM demonstrating a role for Fuc mediated binding events in this important process ${ }^{151}$. More recent MALDI-TOF mass spectrometry analyses of CD11b/ CD18 purified from human PMN revealed the total profile of $\mathrm{N}$-linked glycans on both the unique $\mathrm{CD} 11 \mathrm{~b}$ and the common CD18 integrin subunits. Glycan analysis of CD11b highlighted an unusual lack of sialylation, an over-abundance of high Man glycans (including the binding target of the macrophage Man receptor) along with the expression of unusual bisected GlcNAc glycan structures ${ }^{152}$. Importantly PHA-E lectin targeting of bisected GlcNAc N-glycans on CD11b deactivated PMN Syk signaling, reduced levels of PMN TEpM, decreased reactive oxygen species release and increased rates of PMN phagocytosis and apoptosis $^{152}$.

Targeting of the type 1 Lewis family member Lewis A [Gal $\beta 1-3$ (Fuc a1-4)GlcNAc-R, Le ${ }^{a}$ ] on PMN has been shown to increase levels of $\mathrm{TEPM}^{153}$. Interestingly, in contrast to effects seen downstream of $\mathrm{Le}^{\mathrm{a}}$ engagement, targeting of the related type 2 Lewis glycan structure Lewis X [Galß1-4(Fuca1-3)GIcNAc-R, Le ${ }^{x}$, expressed on $C D 11 \mathrm{~b} / \mathrm{CD} 18$, reduced human PMN TEpM, decreased trafficking of murine PMN to the proximal colon and increased PMN phagocytosis and degranulation responses ${ }^{152,154}$. Taken together, these studies highlight that targeting specific Lewis family glycan structures results in contrasting effects on key PMN inflammatory effector functions suggesting that these glycans could represent rational targets for ameliorating inappropriate PMN-intestinal influx and activation during IBDassociated intestinal inflammation.

In addition to Lewis family glycans, recent literature has established that azurophilic granule proteins from human PMN (including myeloperoxidase, cathepsin G, proteinase 3, azurocidin and elastase) display unconventional glycans that lack Man residues (paucimannosidic glycans) and monoantennary (single branched) complex type $\mathrm{N}$-glycoproteins infrequently reported in other areas of human glycobiology ${ }^{155-160}$. Functional studies have revealed that truncated paucimannosidic $\mathrm{N}$-glycans may be more accessible than conventional high Man N-glycans which could result in enhanced affinity of PMN glycoproteins (including elastase) to ligands such as Man-binding lectins and a1antitrypsin that are expressed in inflamed tissues ${ }^{161}$. Indeed, MPO-specific glycan analyses have confirmed the presence of truncated paucimannosidic N-glycans and revealed site-specific glycosylation of PMN MPO with uncommon high Man glycan structures $^{162}$. While the role of paucimannosidic or high Man glycans in modulating pro-inflammatory effects of MPO in intestinal mucosa have yet to be determined, it is likely that atypical glycans on PMN MPO may promote MPO clearance from sites of inflammation by macrophages or dendritic cells that express specific Man-binding lectins ${ }^{163}$.

A recent human PMN granule mapping study elegantly highlighted that glycan complexity increases progressively during PMN granulopoiesis, suggesting temporal changes in the PMN glycosylation machinery resulting in granule-specific glycan signatures $^{164}$. Unusual truncated paucimannosidic N-glycans and a complete lack of O-glycosylation on glycoproteins were found within human PMN azurophilic granules. This uncommon deficit of $O$ glycosylation suggests that azurophilic granules are formed at a stage of granulopoiesis where a-GalNAc-transferases are not actively expressed. Like azurophilic granules, specific and gelatinase containing granules were shown to display rare glycan signatures including complex $\mathrm{N}$ - and O-glycans with unusually elongated poly- $N$-acetyllactosamine (poly-LacNAc) repeats decorated with Lewis glycans. These long poly-LacNAc extensions have not yet been identified on other cells, highlighting unconventional biosynthetic pathways in PMN that likely underpin cell-specific glycosylation features and PMN specific functional properties. Long poly-LacNAc repeats could promote binding interactions between PMN and multivalent Galectins (Gal binding lectins) that are released into the gut lumen during inflammation ${ }^{165,166}$. Along with effects on PMN trafficking, phagocytosis, and degranulation it has become increasingly apparent that glycans modulate the formation and activity of neutrophil extracellular traps (NETs) ${ }^{167}$. Human and bovine PMN express polySia glycans (extended chains of a2-8-linked Sia) that can modulate the binding of lactoferrin to NETs to decrease overall levels of NETosis ${ }^{168,169}$. Similarly, reduced ionomycin and PMA induced NETosis was reported downstream of PMN binding to highly glycosylated mucins ${ }^{170}$.

To date, studies of PMN glycosylation have highlighted several important concepts including cell, site, and protein specific glycosylation. Therefore, it can be speculated that discrete PMN glycans bind to specific GBPs triggering differential regulation of inflammatory effector functions in inflamed mucosal tissues. Extracellular glycan chains terminate with a limited number of specific terminal monosaccharides. Common capping features of glycan chains including GlcNAcylation, galactosylation, fucosylation, and sialylation form the recognition motifs for GBPs which contain one or more carbohydrate recognition domains that bind specific configurations of 2-7 monosaccharide residues ${ }^{171}$. Studies have shown that PMN spatiotemporally display a wide variety of GBPs including Galectins, Siglecs, Man recognizing lectins, and glycan-binding integrins that shape PMN function with remarkable plasticity ${ }^{172}$. Furthermore, given the relatively short half-life and lack of robust transcriptional activity of these GBPs, it may be speculated that mature PMN have evolved to use glycan remodeling as a rapid way to fine tune critical functional responses. As such terminal glycans may be a rational target for regulating $\mathrm{PMN}$ trafficking and modulating effector functions in inflammatory diseases where dysregulated PMN influx is associated with mucosal tissue damage.

\section{T-CELL GLYCOSYLATION CONTRIBUTES TO INTESTINAL INFLAMMATION}

In addition to innate immune cells, uncontrolled activation of adaptive immune mediators including $T$ cells plays a key role in the pathogenesis of IBD. Critically, altered T-cell glycosylation is directly implicated in the pathobiology of IBD. Lamina propria $\mathrm{T}$ cells isolated from people with UC display decreased $\mathrm{N}$-acetylglucosaminyltransferase $\mathrm{V}$ (MGAT5) activity resulting in reduced levels of $\beta 1-6$ branched surface glycans (Fig. 3). Furthermore, lower levels of $\beta 1-6$ T-cell glycosylation was found to correlate with increased IBD severity ${ }^{173,174}$. A recent study has demonstrated that low levels of branched $\mathrm{N}$-glycans detected on $\mathrm{T}$ cells around the time of UC diagnosis is predictive of poor response to standard IBD therapies indicating the likelihood of a more severe course of disease in these individuals ${ }^{175}$. Deficiency of $\beta 1-6$ branching on N-glycans in $\mathrm{Mgat5}^{-1-}$ mice has been shown to promote T-cell receptor (TCR) clustering, decrease TCR activation thresholds, and increase T-cell activation leading to increased susceptibility to experimental colitis ${ }^{176}$. Along with changes in glycan branching, increased levels of fucosyltransferase 8 (FUT8) mediated core fucosylation is observed in intestinal T cells isolated from inflamed mucosa of UC and Crohn's disease patients with augmented fucosylation correlating with higher endoscopic disease activity scores ${ }^{177}$. Similarly, increased core fucosylation has been observed in mucosal $T$ cells from colitic mice while Fut8 deficiency protects from development of TNBS mediated colitis. Functionally, it was shown that $\mathrm{CD} 4^{+} \mathrm{T}$ cells from Fut $^{-/-}$mice have reduced pro-inflammatory cytokine production suggesting that core fucosylation of TCRs is required for T-cell signaling and induction of colitis in vivo ${ }^{177}$. 
In addition to Fuc-containing glycans, T cells express LacNAc polymers that display the glycan ligands for numerous Galectins. Galectin-2 has been shown to induce apoptosis of mucosal T cells in mice and ameliorate DSS and T-cell transfer induced colitis in vivo ${ }^{178}$. Another ubiquitously expressed Galectin, Galectin-3 is known to form a molecular lattice between Gal glycans on TCRs and N-glycans on the phosphatase CD45 that inhibits downstream T-cell activation ${ }^{179,180}$. Furthermore, knockout of Galectin-3 in mice resulted in more severe DSS-induced colitis, while supplementation of wild-type mice with Galectin-3 reduced disease severity through induction of regulatory $T$ cells in the intestinal mucosa ${ }^{181}$. Importantly, there is reduced Galectin-3 expression in the inflamed intestinal epithelium of IBD patients suggesting that altered Galectin-3-T-cell signaling interactions may contribute to human disease pathobiology ${ }^{182,183}$. Similar to anti-inflammatory effects observed with Galectin-3, administration of Galectin-1 to mice before induction of colitis has been shown to reduce the number of hapten activated splenic T cells, decrease production of INFY by intestinal T cells and reduce overall severity of disease ${ }^{184}$. In contrast to protective effects of Galectins-1-3 on T-cell function during colitis, the epithelial cell lectin, Galectin- 4 has been shown to stimulate IL- 6 production by mucosal $\mathrm{CD}^{+}{ }^{+} \mathrm{T}$ cells, and delay recovery from inflammation-induced colonic injury ${ }^{185}$. Further investigations are clearly needed to fully understand how glycosylation regulates mucosal T-cell function. However, studies to date highlight how alterations in T-cell glycosylation represent potentially diagnostic IBD biomarkers as well as druggable targets for reduction of chronic intestinal inflammatory responses.

\section{CONCLUSION/SUMMARY}

The enormous diversity of mammalian glycans $(>15,000$ specific glycan determinants identified to date ${ }^{186}$ ) coupled with the high density of cell surface glycosylation makes uncovering the biological functions of individual glycan structures extremely challenging. However, the role glycosylation plays in driving mucosal pathogenesis under conditions of chronic inflammation is an exciting emerging field with multiple directions for future research. Work to date has highlighted that inflammation induced changes in intestinal and immune cell glycosylation produce potentially prognostic biomarkers in addition to generating targets for development of new therapeutic strategies for amelioration of IBD-associated mucosal tissue damage. One of the main challenges for the future will be determining how different biological contexts can impart cell and protein-specific glycan encoded signals to regulate mucosal homeostasis and disease. With the advent of exciting new analytical technologies including Surface-protein Glycan and RNA-seq, a recently developed method that facilitates analysis of $\mathrm{N}$-linked glycosylation, extracellular epitopes, and the transcriptome at a single cell level ${ }^{187}$, our understanding of the molecular basis of cellspecific glycan mediated signaling during mucosal inflammation is set to improve considerably in the years to come.

\section{REFERENCES}

1. Struwe, W. B. \& Robinson, C. V. Relating glycoprotein structural heterogeneity to function - insights from native mass spectrometry. Curr. Opin. Struct. Biol. 58, 241-248 (2019).

2. Cummings, R. D. et al. (eds). Essentials of Glycobiology (Cold Spring Harbor, NY, 2015), pp 373-385.

3. Seeberger P. H. Monosaccharide diversity. In Essentials of Glycobiology (ed Varki, A. et al.) 19-30 (Cold Spring Harbor, NY, 2015).

4. Nakamura, K. et al. Interspecies comparison of muscle gangliosides by twodimensional thin-layer chromatography. J. Biochem. 94, 1359-1365 (1983).

5. Nairn, A. V. et al. Regulation of glycan structures in animal tissues: transcript profiling of glycan-related genes. J. Biol. Chem. 283, 17298-17313 (2008).

6. Stanley, P. et al. (eds). Essentials of Glycobiology (Cold Spring Harbor, NY, 2015), pp 99-111.
7. Brockhausen, I., Stanley, P. O-GaINAc Glycans. In Essentials of Glycobiology (eds Varki A, Cummings, RD, Esko, JD, Stanley, P, Hart, GW et al.) 113-123 (Cold Spring Harbor, NY, 2015), pp 113-123.

8. Ju, T., Brewer, K., D'Souza, A., Cummings, R. D. \& Canfield, W. M. Cloning and expression of human core 1 beta1,3-galactosyltransferase. J. Biol. Chem. 277, 178-186 (2002).

9. Sun, X., Zhan, M., Sun, X., Liu, W. \& Meng, X. C1GALT1 in health and disease. Oncol. Lett. 22, 589 (2021).

10. Iwai, T. et al. Molecular cloning and characterization of a novel UDP-GlcNAc: GalNAc-peptide beta1,3-N-acetylglucosaminyltransferase (beta 3Gn-T6), an enzyme synthesizing the core 3 structure of O-glycans. J. Biol. Chem. 277, 12802-12809 (2002).

11. Iwai, T. et al. Core 3 synthase is down-regulated in colon carcinoma and profoundly suppresses the metastatic potential of carcinoma cells. Proc. Natl Acad. Sci. USA 102, 4572-4577 (2005).

12. Bierhuizen, M. F. \& Fukuda, M. Expression cloning of a cDNA encoding UDPGIcNAc:Gal beta 1-3-GalNAc-R (GIcNAc to GalNAc) beta 1-6GlcNAc transferase by gene transfer into $\mathrm{CHO}$ cells expressing polyoma large tumor antigen. Proc. Natl Acad. Sci. USA 89, 9326-9330 (1992).

13. Yeh, J. C., Ong, E. \& Fukuda, M. Molecular cloning and expression of a novel beta-1, 6-N-acetylglucosaminyltransferase that forms core 2, core 4, and I branches. J. Biol. Chem. 274, 3215-3221 (1999).

14. An, G. et al. Increased susceptibility to colitis and colorectal tumors in mice lacking core 3-derived O-glycans. J. Exp. Med. 204, 1417-1429 (2007).

15. Fu, J. et al. Loss of intestinal core 1-derived O-glycans causes spontaneous colitis in mice. J. Clin. Investig. 121, 1657-1666 (2011).

16. Ju, T. \& Cummings, R. D. A unique molecular chaperone Cosmc required for activity of the mammalian core 1 beta 3-galactosyltransferase. Proc. Natl Acad. Sci. USA 99, 16613-16618 (2002).

17. Robbe, C. et al. Evidence of regio-specific glycosylation in human intestinal mucins: presence of an acidic gradient along the intestinal tract. J. Biol. Chem. 278, 46337-46348 (2003).

18. Robbe, C., Capon, C., Coddeville, B. \& Michalski, J. C. Structural diversity and specific distribution of $\mathrm{O}-$ glycans in normal human mucins along the intestinal tract. Biochem. J. 384, 307-316 (2004).

19. Robbe-Masselot, $C$. et al. Expression of a core 3 disialyl-Le( $(x)$ hexasaccharide in human colorectal cancers: a potential marker of malignant transformation in colon. J. Proteome Res. 8, 702-711 (2009).

20. Tailford, L. E., Crost, E. H., Kavanaugh, D. \& Juge, N. Mucin glycan foraging in the human gut microbiome. Front. Genet. 6, 81 (2015).

21. Robbe-Masselot, C., Maes, E., Rousset, M., Michalski, J. C. \& Capon, C. Glycosylation of human fetal mucins: a similar repertoire of O-glycans along the intestinal tract. Glycoconj. J. 26, 397-413 (2009).

22. Arike, L., Holmen-Larsson, J. \& Hansson, G. C. Intestinal Muc2 mucin O-glycosylation is affected by microbiota and regulated by differential expression of glycosyltranferases. Glycobiology 27, 318-328 (2017).

23. Holmen Larsson, J. M., Thomsson, K. A., Rodriguez-Pineiro, A. M., Karlsson, H. \& Hansson, G. C. Studies of mucus in mouse stomach, small intestine, and colon. III. Gastrointestinal Muc5ac and Muc2 mucin O-glycan patterns reveal a regiospecific distribution. Am. J. Physiol. Gastrointest. Liver Physiol. 305, G357-G363 (2013).

24. Allavena, P. et al. Engagement of the mannose receptor by tumoral mucins activates an immune suppressive phenotype in human tumor-associated macrophages. Clin. Dev. Immunol. 2010, 547179 (2010).

25. Abed, J. et al. Fap2 mediates Fusobacterium nucleatum colorectal adenocarcinoma enrichment by binding to tumor-expressed Gal-GalNAc. Cell Host Microbe 20, 215-225 (2016)

26. Shan, M. et al. Mucus enhances gut homeostasis and oral tolerance by delivering immunoregulatory signals. Science 342, 447-453 (2013).

27. Sonnenburg, J. L. et al. Glycan foraging in vivo by an intestine-adapted bacterial symbiont. Science 307, 1955-1959 (2005).

28. Goto, Y. et al. IL-10-producing CD4(+) T cells negatively regulate fucosylation of epithelial cells in the gut. Sci. Rep. 5, 15918 (2015).

29. Goto, Y. et al. Innate lymphoid cells regulate intestinal epithelial cell glycosylation. Science 345, 1254009 (2014).

30. Kostopoulos, I. et al. A continuous battle for host-derived glycans between a mucus specialist and a glycan generalist in vitro and in vivo. Front. Microbiol. 12, 632454 (2021).

31. Jiang, $H$. et al. Modulating cell-surface receptor signaling and ion channel functions by in situ glycan editing. Angew. Chem. Int. Ed. Engl. 57, 967-971 (2018).

32. Rouhanifard, S. H., Lopez Aguilar, A., Meng, L., Moremen, K. W. \& Wu, P. Engineered glycocalyx regulates stem cell proliferation in murine crypt organoids. Cell Chem. Biol. 25, 439-446 e435 (2018). 
33. Kelm M., et al. Targeting epithelium-expressed sialyl Lewis glycans improves colonic mucosal wound healing and protects against colitis. JCl Insight. 5, e135843 (2020)

34. Brazil, J. C. et al. alpha3/4 Fucosyltransferase 3-dependent synthesis of Sialyl Lewis A on CD44 variant containing exon 6 mediates polymorphonuclear leukocyte detachment from intestinal epithelium during transepithelial migration. J. Immunol. 191, 4804-4817 (2013).

35. Kudelka, M. R. et al. Cosmc is an X-linked inflammatory bowel disease risk gene that spatially regulates gut microbiota and contributes to sex-specific risk. Proc. Natl Acad. Sci. USA 113, 14787-14792 (2016).

36. McGovern, D. P. et al. Fucosyltransferase 2 (FUT2) non-secretor status is associated with Crohn's disease. Hum. Mol. Genet. 19, 3468-3476 (2010).

37. Rausch, P. et al. Colonic mucosa-associated microbiota is influenced by an interaction of Crohn disease and FUT2 (Secretor) genotype. Proc. Natl Acad. Sci. USA 108, 19030-19035 (2011).

38. Suzuki, K. et al. Intestinal epithelial cell-specific deletion of alpha-mannosidase II ameliorates experimental colitis. Cell Struct. Funct. 43, 25-39 (2018).

39. Lauc, G. et al. Loci associated with N-glycosylation of human immunoglobulin $\mathrm{G}$ show pleiotropy with autoimmune diseases and haematological cancers. PLOS Genet 9, e1003225 (2013).

40. Theodoratou, E. et al. The role of glycosylation in IBD. Nat. Rev. Gastroenterol. Hepatol. 11, 588-600 (2014).

41. Momozawa, Y. et al. IBD risk loci are enriched in multigenic regulatory modules encompassing putative causative genes. Nat. Commun. 9, 2427 (2018).

42. Camacho, F. I. et al. CD44v6 expression in inflammatory bowel disease is associated with activity detected by endoscopy and pathological features. Histopathology 35, 144-149 (1999).

43. Reinisch, W., Heider, K. H., Oberhuber, G., Dejaco, C. \& Adolf, G. R. Expression of CD44v6 in ulcerative colitis and Crohn's disease. Lancet 347, 1049 (1996).

44. Huang, Y. L., Chassard, C., Hausmann, M., von Itzstein, M. \& Hennet, T. Sialic acid catabolism drives intestinal inflammation and microbial dysbiosis in mice. Nat. Commun. 6, 8141 (2015).

45. Moore, R., King, N. \& Alroy, J. Differences in cellular glycoconjugates of quiescent, inflamed, and neoplastic colonic epithelium in colitis and cancer-prone tamarins. Am. J. Pathol. 131, 484-489 (1988).

46. Boland, C. R. \& Clapp, N. K. Glycoconjugates in the colons of New World monkeys with spontaneous colitis. Association between inflammation and neoplasia. Gastroenterology 92, 625-634 (1987).

47. Hounsell, E. F. \& Feizi, T. Gastrointestinal mucins. Structures and antigenicities of their carbohydrate chains in health and disease. Med. Biol. 60, 227-236 (1982).

48. Rhodes, J. M., Black, R. R. \& Savage, A. Altered lectin binding by colonic epithelial glycoconjugates in ulcerative colitis and Crohn's disease. Dig. Dis. Sci. 33, 1359-1363 (1988).

49. Boland, C. R., Lance, P., Levin, B., Riddell, R. H. \& Kim, Y. S. Abnormal goblet cell glycoconjugates in rectal biopsies associated with an increased risk of neoplasia in patients with ulcerative colitis: early results of a prospective study. Gut $\mathbf{2 5}$, 1364-1371 (1984).

50. Campbell, B. J., Finnie, I. A., Hounsell, E. F. \& Rhodes, J. M. Direct demonstration of increased expression of Thomsen-Friedenreich (TF) antigen in colonic adenocarcinoma and ulcerative colitis mucin and its concealment in normal mucin. J. Clin. Investig. 95, 571-576 (1995).

51. Cooper, H. S. Peanut lectin-binding sites in large bowel carcinoma. Lab Investig. 47, 383-390 (1982).

52. Bodger, K. et al. Altered colonic glycoprotein expression in unaffected monozygotic twins of inflammatory bowel disease patients. Gut 55, 973-977 (2006).

53. Ryder, S. D., Smith, J. A. \& Rhodes, J. M. Peanut lectin: a mitogen for normal human colonic epithelium and human HT29 colorectal cancer cells. J. Natl Cancer Inst. 84, 1410-1416 (1992).

54. Ryder, S. D., Jacyna, M. R., Levi, A. J., Rizzi, P. M. \& Rhodes, J. M. Peanut ingestion increases rectal proliferation in individuals with mucosal expression of peanut lectin receptor. Gastroenterology 114, 44-49 (1998)

55. Moulari, B., Beduneau, A., Pellequer, Y. \& Lamprecht, A. Lectin-decorated nanoparticles enhance binding to the inflamed tissue in experimental colitis. J. Control Release 188, 9-17 (2014).

56. Su, W. et al. Fusobacterium nucleatum promotes the development of ulcerative colitis by inducing the autophagic cell death of intestinal epithelial. Front. Cell. Infect. Microbiol. 10, 594806 (2020).

57. Terahara, K. et al. Distinct fucosylation of $M$ cells and epithelial cells by Fut1 and Fut2, respectively, in response to intestinal environmental stress. Biochem. Biophys. Res. Commun. 404, 822-828 (2011).

58. Lin, B. et al. GDP-fucose: beta-galactoside alpha1,2-fucosyltransferase, MFUT-II, and not MFUT-I or -III, is induced in a restricted region of the digestive tract of germ-free mice by host-microbe interactions and cycloheximide. Biochim. Biophys. Acta 1487, 275-285 (2000).
59. Wang, Y. et al. Fucosylation deficiency in mice leads to colitis and adenocarcinoma. Gastroenterology 152, 193-205 e110 (2017).

60. Hurd, E. A., Holmen, J. M., Hansson, G. C. \& Domino, S. E. Gastrointestinal mucins of Fut2-null mice lack terminal fucosylation without affecting colonization by Candida albicans. Glycobiology 15, 1002-1007 (2005).

61. Lagerqvist, A., Hakansson, D., Frank, H., Seidel, A. \& Jenssen, D. Structural requirements for mutation formation from polycyclic aromatic hydrocarbon dihydrodiol epoxides in their interaction with food chemopreventive compounds. Food Chem. Toxicol. 49, 879-886 (2011).

62. Bjork, S., Breimer, M. E., Hansson, G. C., Karlsson, K. A. \& Leffler, H. Structures of blood group glycosphingolipids of human small intestine. A relation between the expression of fucolipids of epithelial cells and the ABO, Le and Se phenotype of the donor. J. Biol. Chem. 262, 6758-6765 (1987).

63. Sakamoto, S. et al. Expression of Lewisa, Lewisb, Lewisx, Lewisy, siayl-Lewisa, and sialyl-Lewisx blood group antigens in human gastric carcinoma and in normal gastric tissue. Cancer Res. 49, 745-752 (1989).

64. Yuan, M. et al. Distribution of blood group antigens $A, B, H$, Lewisa, and Lewisb in human normal, fetal, and malignant colonic tissue. Cancer Res. 45, 4499-4511 (1985).

65. Franke, A. et al. Genome-wide meta-analysis increases to 71 the number of confirmed Crohn's disease susceptibility loci. Nat. Genet. 42, 1118-1125 (2010).

66. $\mathrm{Wu}, \mathrm{H}$. et al. Association of fucosyltransferase 2 gene polymorphisms with inflammatory bowel disease in patients from Southeast China. Gastroenterol. Res Pract. 2017, 4148651 (2017).

67. Parmar, A. S. et al. Association study of FUT2 (rs601338) with celiac disease and inflammatory bowel disease in the Finnish population. Tissue Antigens 80, 488-493 (2012).

68. Hu, D. et al. Association of ulcerative colitis with FUT2 and FUT3 polymorphisms in patients from Southeast China. PLoS ONE 11, e0146557 (2016).

69. Stanley P., Cummings R. D. Structures Common to Different Glycans. In Essentials of Glycobiology. (eds Varki A., Cummings, R. D., Esko, J. D., Stanley, P., Hart, G. W. et al.) 161-178 (Cold Spring Harbor, NY, 2015).

70. Shirato, $\mathrm{H}$. et al. Noroviruses distinguish between type 1 and type 2 histo-blood group antigens for binding. J. Virol. 82, 10756-10767 (2008).

71. Pacheco, A. R. et al. Fucose sensing regulates bacterial intestinal colonization. Nature 492, 113-117 (2012).

72. Pham, T. A. et al. Epithelial IL-22RA1-mediated fucosylation promotes intestinal colonization resistance to an opportunistic pathogen. Cell Host Microbe 16, 504-516 (2014)

73. Miyoshi, J. et al. Ectopic expression of blood type antigens in inflamed mucosa with higher incidence of FUT2 secretor status in colonic Crohn's disease. J. Gastroenterol. 46, 1056-1063 (2011).

74. $\mathrm{Ke}$, J. et al. Fucose ameliorate intestinal inflammation through modulating the crosstalk between bile acids and gut microbiota in a chronic colitis murine model. Inflamm. Bowel Dis. 26, 863-873 (2020).

75. Sitkin, S., Vakhitov, T., Kononova, S., Skalinskaya, M. \& Pokrotnieks, J. Gut microbiota-mediated pleiotropic effects of fucose can improve inflammatory bowel disease by modulating bile acid metabolism and enhancing propionate production. Inflamm. Bowel Dis. 27, e10-e11 (2021).

76. He, R. et al. L-Fucose ameliorates DSS-induced acute colitis via inhibiting macrophage M1 polarization and inhibiting NLRP3 inflammasome and NF-kB activation. Int. Immunopharmacol. 73, 379-388 (2019).

77. Karlen, P. et al. Sialyl-Tn antigen as a marker of colon cancer risk in ulcerative colitis: relation to dysplasia and DNA aneuploidy. Gastroenterology 115, 1395-1404 (1998).

78. Itzkowitz, S. H. et al. Sialosyl-Tn antigen: initial report of a new marker of malignant progression in long-standing ulcerative colitis. Gastroenterology 109, 490-497 (1995)

79. Itzkowitz, S. H. et al. Sialosyl-Tn antigen is prevalent and precedes dysplasia in ulcerative colitis: a retrospective case-control study. Gastroenterology 110 694-704 (1996)

80. Papadogiannakis, N., Gad, A., Chenard, M. P., Bellocq, J. P. \& Duclos, B. Expression of CD44 variants in differential diagnosis of ulcerative colitis and Crohn's disease. Lancet 347, 1413-1414 (1996).

81. Rosenberg, W. M. et al. Increased expression of CD44v6 and CD44v3 in ulcerative colitis but not colonic Crohn's disease. Lancet 345, 1205-1209 (1995).

82. Larsson, J. M. et al. Altered O-glycosylation profile of MUC2 mucin occurs in active ulcerative colitis and is associated with increased inflammation. Inflamm. Bowel Dis. 17, 2299-2307 (2011)

83. Cooper, H. S. \& Steplewski, Z. Immunohistologic study of ulcerative colitis with monoclonal antibodies against tumor-associated and/or differentiation antigens. Gastroenterology 95, 686-693 (1988).

84. Frykholm, G., Enblad, P., Pahlman, L. \& Busch, C. Expression of the carcinomaassociated antigens CA 19-9 and CA-50 in inflammatory bowel disease. Dis. Colon Rectum 30, 545-548 (1987). 
85. Tarris, G. et al. Specific norovirus interaction with Lewis $x$ and Lewis a on human intestinal inflammatory mucosa during refractory inflammatory bowel disease. mSphere 6, e01185-20 (2021).

86. Brazil, J. C. et al. Neutrophil migration across intestinal epithelium: evidence for a role of CD44 in regulating detachment of migrating cells from the luminal surface. J. Immunol. 185, 7026-7036 (2010).

87. Parikh, K. et al. Colonic epithelial cell diversity in health and inflammatory bowel disease. Nature 567, 49-55 (2019).

88. Nystrom, E. E. L. et al. An intercrypt subpopulation of goblet cells is essential for colonic mucus barrier function. Science 372, eabb1590 (2021).

89. Ermund, A., Schutte, A., Johansson, M. E., Gustafsson, J. K. \& Hansson, G. C. Studies of mucus in mouse stomach, small intestine, and colon. I. Gastrointestinal mucus layers have different properties depending on location as well as over the Peyer's patches. Am. J. Physiol. Gastrointest. Liver Physiol. 305, G341-G347 (2013).

90. Johansson, M. E., Larsson, J. M. \& Hansson, G. C. The two mucus layers of colon are organized by the MUC2 mucin, whereas the outer layer is a legislator of host-microbial interactions. Proc. Natl Acad. Sci. USA 108, 4659-4665 (2011).

91. Hollingsworth, M. A. \& Swanson, B. J. Mucins in cancer: protection and control of the cell surface. Nat. Rev. Cancer 4, 45-60 (2004).

92. Johansson, M. E. et al. The inner of the two Muc2 mucin-dependent mucus layers in colon is devoid of bacteria. Proc. Natl Acad. Sci. USA 105, 15064-15069 (2008).

93. Louis, P., Scott, K. P., Duncan, S. H. \& Flint, H. J. Understanding the effects of diet on bacterial metabolism in the large intestine. J. Appl. Microbiol. 102, 1197-1208 (2007).

94. Lee, S. M. et al. Bacterial colonization factors control specificity and stability of the gut microbiota. Nature 501, 426-429 (2013).

95. Tobisawa, Y., Imai, Y., Fukuda, M. \& Kawashima, H. Sulfation of colonic mucins by $\mathrm{N}$-acetylglucosamine 6 -O-sulfotransferase- 2 and its protective function in experimental colitis in mice. J. Biol. Chem. 285, 6750-6760 (2010).

96. Dawson, P. A. et al. Reduced mucin sulfonation and impaired intestinal barrier function in the hyposulfataemic NaS1 null mouse. Gut 58, 910-919 (2009).

97. Corfield, A. P. et al. Colonic mucins in ulcerative colitis: evidence for loss of sulfation. Glycoconj. J. 13, 809-822 (1996).

98. Van Klinken, B. J., Van der Wal, J. W., Einerhand, A. W., Buller, H. A. \& Dekker, J. Sulphation and secretion of the predominant secretory human colonic mucin MUC2 in ulcerative colitis. Gut 44, 387-393 (1999).

99. Raouf, A. H. et al. Sulphation of colonic and rectal mucin in inflammatory bowel disease: reduced sulphation of rectal mucus in ulcerative colitis. Clin. Sci. 83, 623-626 (1992).

100. Tsai, H. H., Dwarakanath, A. D., Hart, C. A., Milton, J. D. \& Rhodes, J. M. Increased faecal mucin sulphatase activity in ulcerative colitis: a potential target for treatment. Gut 36, 570-576 (1995).

101. Probert, C. S. et al. South Asian and European colitics show characteristic differences in colonic mucus glycoprotein type and turnover. Gut 36, 696-702 (1995)

102. Foster, A. \& Jacobson, K. Changing incidence of inflammatory bowel disease: environmental influences and lessons learnt from the South asian population. Front. Pediatr. 1, 34 (2013).

103. Macauley, M. S., Crocker, P. R. \& Paulson, J. C. Siglec-mediated regulation of immune cell function in disease. Nat. Rev. Immunol. 14, 653-666 (2014).

104. von Gunten, S. et al. Siglec-9 transduces apoptotic and nonapoptotic death signals into neutrophils depending on the proinflammatory cytokine environment. Blood 106, 1423-1431 (2005).

105. McMillan, S. J. et al. Siglec-E is a negative regulator of acute pulmonary neutrophil inflammation and suppresses CD11b beta2-integrin-dependent signaling. Blood 121, 2084-2094 (2013)

106. McMillan, S. J., Sharma, R. S., Richards, H. E., Hegde, V. \& Crocker, P. R. Siglec-E promotes beta2-integrin-dependent NADPH oxidase activation to suppress neutrophil recruitment to the lung. J. Biol. Chem. 289, 20370-20376 (2014).

107. Bergstrom, K. et al. Defective intestinal mucin-type O-glycosylation causes spontaneous colitis-associated cancer in mice. Gastroenterology 151, 152-164 e111 (2016).

108. Wang, Y. et al. Cosmc is an essential chaperone for correct protein O-glycosylation. Proc. Natl Acad. Sci. USA 107, 9228-9233 (2010).

109. Chang, D. et al. Accounting for eXentricities: analysis of the $X$ chromosome in GWAS reveals $X$-linked genes implicated in autoimmune diseases. PLOS ONE 9, e113684 (2014).

110. Kaneko, Y., Nimmerjahn, F. \& Ravetch, J. V. Anti-inflammatory activity of immunoglobulin G resulting from Fc sialylation. Science 313, 670-673 (2006).

111. Pucic, M. et al. High throughput isolation and glycosylation analysis of IgGvariability and heritability of the IgG glycome in three isolated human populations. Mol. Cell Proteom. 10, M111 010090 (2011).
112. Simurina, M. et al. Glycosylation of immunoglobulin $G$ associates with clinical features of inflammatory bowel diseases. Gastroenterology 154, 1320-1333 e1310 (2018).

113. Trbojevic Akmacic, I. et al. Inflammatory bowel disease associates with proinflammatory potential of the immunoglobulin G glycome. Inflamm. Bowel Dis. 21, 1237-1247 (2015).

114. Castro-Dopico, T. \& Clatworthy, M. R. Mucosal IgG in inflammatory bowel disease - a question of (sub)class? Gut Microbes 12, 1-9 (2020).

115. Kappler, K., Lasanajak, Y., Smith, D. F., Opitz, L. \& Hennet, T. Increased antibody response to fucosylated oligosaccharides and fucose-carrying bacteroides species in Crohn's disease. Front. Microbiol. 11, 1553 (2020).

116. Chakraborty, S. et al. Proinflammatory lgG Fc structures in patients with severe COVID-19. Nat. Immunol. 22, 67-73 (2021).

117. Dube, R. et al. Agalactosyl lgG in inflammatory bowel disease: correlation with C-reactive protein. Gut 31, 431-434 (1990).

118. Nakajima, S. et al. Functional analysis of agalactosyl IgG in inflammatory bowel disease patients. Inflamm. Bowel Dis. 17, 927-936 (2011).

119. Shinzaki, S. et al. IgG oligosaccharide alterations are a novel diagnostic marker for disease activity and the clinical course of inflammatory bowel disease. Am. J. Gastroenterol. 103, 1173-1181 (2008).

120. Parekh, R. B. et al. Galactosylation of IgG associated oligosaccharides: reduction in patients with adult and juvenile onset rheumatoid arthritis and relation to disease activity. Lancet 1, 966-969 (1988).

121. Choteau, L. et al. Role of mannose-binding lectin in intestinal homeostasis and fungal elimination. Mucosal Immunol. 9, 767-776 (2016).

122. Chang, S., Malter, L. \& Hudesman, D. Disease monitoring in inflammatory bowel disease. World J. Gastroenterol. 21, 11246-11259 (2015).

123. Panes, J., Jairath, V. \& Levesque, B. G. Advances in use of endoscopy, radiology, and biomarkers to monitor inflammatory bowel diseases. Gastroenterology 152, 362-373 e363 (2017).

124. Springer, G. F., Williamson, P. \& Brandes, W. C. Blood group activity of gramnegative bacteria. J. Exp. Med. 113, 1077-1093 (1961).

125. Yilmaz, B. et al. Gut microbiota elicits a protective immune response against malaria transmission. Cell 159, 1277-1289 (2014).

126. Bello-Gil, D. et al. The formation of glycan-specific natural antibodies repertoire in GalT-KO mice is determined by gut microbiota. Front. Immunol. 10, 342 (2019).

127. Dotan, I. et al. Antibodies against laminaribioside and chitobioside are novel serologic markers in Crohn's disease. Gastroenterology 131, 366-378 (2006).

128. Rieder, F. et al. Association of the novel serologic anti-glycan antibodies antilaminarin and anti-chitin with complicated Crohn's disease behavior. Inflamm. Bowel Dis. 16, 263-274 (2010).

129. Paul, S. et al. Association of anti-glycan antibodies and inflammatory bowel disease course. J. Crohns Colitis 9, 445-451 (2015).

130. Martin, H. M. et al. Enhanced Escherichia coli adherence and invasion in Crohn's disease and colon cancer. Gastroenterology 127, 80-93 (2004).

131. Ott, S. J. et al. Reduction in diversity of the colonic mucosa associated bacterial microflora in patients with active inflammatory bowel disease. Gut 53, 685-693 (2004).

132. Manichanh, C. et al. Reduced diversity of faecal microbiota in Crohn's disease revealed by a metagenomic approach. Gut 55, 205-211 (2006).

133. Day, C. J. et al. Glycan:glycan interactions: high affinity biomolecular interactions that can mediate binding of pathogenic bacteria to host cells. Proc. Natl Acad. Sci. USA 112, E7266-E7275 (2015).

134. Mubaiwa, T. D. et al. The glycointeractome of serogroup B Neisseria meningitidis strain MC58. Sci. Rep. 7, 5693 (2017).

135. Brazil, J. C. \& Parkos, C. A. Pathobiology of neutrophil-epithelial interactions. Immunol. Rev. 273, 94-111 (2016).

136. Brazil, J. C., Quiros, M., Nusrat, A. \& Parkos, C. A. Innate immune cell-epithelial crosstalk during wound repair. J. Clin. Investig. 129, 2983-2993 (2019).

137. Saverymuttu, S. H., Peters, A. M., Lavender, J. P., Chadwick, V. S. \& Hodgson, H. J. In vivo assessment of granulocyte migration to diseased bowel in Crohn's disease. Gut 26, 378-383 (1985)

138. Minar, P. et al. Utility of neutrophil Fcgamma receptor I (CD64) index as a biomarker for mucosal inflammation in pediatric Crohn's disease. Inflamm. Bowel Dis. 20, 1037-1048 (2014).

139. Therrien, A. et al. Recruitment of activated neutrophils correlates with disease severity in adult Crohn's disease. Clin. Exp. Immunol. 195, 251-264 (2019).

140. Kucharzik, T., Walsh, S. V., Chen, J., Parkos, C. A. \& Nusrat, A. Neutrophil transmigration in inflammatory bowel disease is associated with differential expression of epithelial intercellular junction proteins. Am. J. Pathol. 159, 2001-2009 (2001)

141. Laroux, F. S., Pavlick, K. P., Wolf, R. E. \& Grisham, M. B. Dysregulation of intestinal mucosal immunity: implications in inflammatory bowel disease. $N$ Physiol. Sci. 16, 272-277 (2001). 
142. Rosen, S. D. \& Bertozzi, C. R. The selectins and their ligands. Curr. Opin. Cell Biol. 6, 663-673 (1994)

143. Ley, K., Laudanna, C., Cybulsky, M. I. \& Nourshargh, S. Getting to the site of inflammation: the leukocyte adhesion cascade updated. Nat. Rev. Immunol. 7, 678-689 (2007).

144. Yago, T. et al. Core 1-derived O-glycans are essential E-selectin ligands on neutrophils. Proc. Natl Acad. Sci. USA 107, 9204-9209 (2010).

145. Kaila, N. \& BEt, Thomas Design and synthesis of sialyl Lewis $(\mathrm{x})$ mimics as $\mathrm{E}-$ and P-selectin inhibitors. Med. Res. Rev. 22, 566-601 (2002).

146. Katayama, Y., Hidalgo, A., Chang, J., Peired, A. \& Frenette, P. S. CD44 is a physiological E-selectin ligand on neutrophils. J. Exp. Med. 201, 1183-1189 (2005).

147. Bloom, J. W., Madanat, M. S. \& Ray, M. K. Cell line and site specific comparative analysis of the $\mathrm{N}$-linked oligosaccharides on human ICAM-1des454-532 by electrospray ionization mass spectrometry. Biochemistry 35, 1856-1864 (1996).

148. Babu, P. et al. Structural characterisation of neutrophil glycans by ultra sensitive mass spectrometric glycomics methodology. Glycoconj. J. 26, 975-986 (2009).

149. Wilkins, P. P., Moore, K. L., McEver, R. P. \& Cummings, R. D. Tyrosine sulfation of P-selectin glycoprotein ligand-1 is required for high affinity binding to P-selectin. J. Biol. Chem. 270, 22677-22680 (1995).

150. Zen, K., Cui, L. B., Zhang, C. Y. \& Liu, Y. Critical role of mac-1 sialyl lewis $x$ moieties in regulating neutrophil degranulation and transmigration. J. Mol. Biol. 374 54-63 (2007).

151. Colgan, S. P. et al. Receptors involved in carbohydrate binding modulate intestinal epithelial-neutrophil interactions. J. Biol. Chem. 270, 10531-10539 (1995).

152. Kelm, M. et al. Regulation of neutrophil function by selective targeting of glycan epitopes expressed on the integrin CD11b/CD18. FASEB J. 34, 2326-2343 (2020).

153. Brazil, J. C. et al. Expression of Lewis-a glycans on polymorphonuclear leukocytes augments function by increasing transmigration. J. Leukoc. Biol. 102, 753-762 (2017).

154. Brazil, J. C., Sumagin, R., Cummings, R. D., Louis, N. A. \& Parkos, C. A. Targeting of neutrophil Lewis $X$ Blocks transepithelial migration and increases phagocytosis and degranulation. Am. J. Pathol. 186, 297-311 (2016).

155. Thaysen-Andersen, $M$. et al. Human neutrophils secrete bioactive paucimannosidic proteins from azurophilic granules into pathogen-infected sputum. J. Biol. Chem. 290, 8789-8802 (2015).

156. Loke, I., Kolarich, D., Packer, N. H. \& Thaysen-Andersen, M. Emerging roles of protein mannosylation in inflammation and infection. Mol. Asp. Med. 51, 31-55 (2016).

157. Loke, I., Packer, N. H. \& Thaysen-Andersen, M. Complementary LC-MS/MS-based $\mathrm{N}$-glycan, $\mathrm{N}$-glycopeptide, anD Intact $\mathrm{N}$-glycoprotein profiling reveals unconventional Asn71-glycosylation of human neutrophil cathepsin G. Biomolecules 5, 1832-1854 (2015)

158. Olczak, M. \& Watorek, W. Structural analysis of N-glycans from human neutrophil azurocidin. Biochem Biophys. Res. Commun. 293, 213-219 (2002).

159. Ravnsborg, T., Houen, G. \& Hojrup, P. The glycosylation of myeloperoxidase. Biochim. Biophys. Acta 1804, 2046-2053 (2010).

160. Zoega, M., Ravnsborg, T., Hojrup, P., Houen, G. \& Schou, C. Proteinase 3 carries small unusual carbohydrates and associates with alphalpha-defensins. J. Proteom. 75, 1472-1485 (2012).

161. Loke, I., Ostergaard, O., Heegaard, N. H. H., Packer, N. H. \& Thaysen-Andersen, M. Paucimannose-Rich N-glycosylation of Spatiotemporally Regulated Human Neutrophil Elastase Modulates Its Immune Functions. Mol. Cell Proteom. 16, 1507-1527 (2017)

162. Reiding, K. R. et al. Neutrophil myeloperoxidase harbors distinct site-specific peculiarities in its glycosylation. J. Biol. Chem. 294, 20233-20245 (2019).

163. East, L. \& Isacke, C. M. The mannose receptor family. Biochim. Biophys. Acta 1572, 364-386 (2002).

164. Venkatakrishnan, V. et al. Glycan analysis of human neutrophil granules implicates a maturation-dependent glycosylation machinery. J. Biol. Chem. 295, 12648-12660 (2020).

165. Papa Gobbi, R. et al. A galectin-specific signature in the gut delineates Crohn's disease and ulcerative colitis from other human inflammatory intestinal disorders. Biofactors 42, 93-105 (2016).

166. Sundblad, V. et al. Galectins in intestinal inflammation: galectin-1 expression delineates response to treatment in celiac disease patients. Front. Immunol. 9 379 (2018).

167. Bornhofft, K. F. \& Galuska, S. P. Glycans as modulators for the formation and functional properties of neutrophil extracellular traps: used by the forces of good and evil. Front. Immunol. 10, 959 (2019).

168. Kuhnle A., Lutteke T., Bornhofft K. F., Galuska S. P. Polysialic acid modulates the binding of external lactoferrin in neutrophil extracellular traps. Biology 8, 20 (2019).

169. Okubo, K. et al. Lactoferrin suppresses neutrophil extracellular traps release in inflammation. EBioMedicine 10, 204-215 (2016).
170. Bornhofft, K. F. et al. Sialylated cervical mucins inhibit the activation of neutrophils to form neutrophil extracellular traps in bovine in vitro model. Front. Immunol. 10, 2478 (2019).

171. Taylor, M. E. \& Drickamer, K. Convergent and divergent mechanisms of sugar recognition across kingdoms. Curr. Opin. Struct. Biol. 28, 14-22 (2014).

172. Ugonotti J., Chatterjee S., Thaysen-Andersen M. Structural and functional diversity of neutrophil glycosylation in innate immunity and related disorders. Mol Aspects Med. 100882 (2020).

173. Dias, A. M. et al. Dysregulation of T cell receptor N-glycosylation: a molecular mechanism involved in ulcerative colitis. Hum. Mol. Genet. 23, 2416-2427 (2014).

174. Dias, A. M. et al. Metabolic control of T cell immune response through glycans in inflammatory bowel disease. Proc. Natl Acad. Sci. USA 115, E4651-E4660 (2018).

175. Pereira, M. S. et al. A [Glyco]biomarker thaT Predicts Failure to Standard Therapy in Ulcerative Colitis Patients. J. Crohns Colitis 13, 39-49 (2019).

176. Demetriou, M., Granovsky, M., Quaggin, S. \& Dennis, J. W. Negative regulation of T-cell activation and autoimmunity by Mgat5 N-glycosylation. Nature 409, 733-739 (2001).

177. Fujii, H. et al. Core fucosylation on $\mathrm{T}$ cells, required for activation of T-Cell receptor signaling and induction of colitis in mice, is increased in patients with inflammatory bowel disease. Gastroenterology 150, 1620-1632 (2016).

178. Paclik, D. et al. Galectin-2 induces apoptosis of lamina propria T lymphocytes and ameliorates acute and chronic experimental colitis in mice. J. Mol. Med. 86, 1395-1406 (2008)

179. Wolfert, M. A. \& Boons, G. J. Adaptive immune activation: glycosylation does matter. Nat. Chem. Biol. 9, 776-784 (2013).

180. Hermiston, M. L., Xu, Z. \& Weiss, A. CD45: a critical regulator of signaling thresholds in immune cells. Annu Rev. Immunol. 21, 107-137 (2003).

181. Tsai, H. F. et al. Galectin-3 suppresses mucosal inflammation and reduces disease severity in experimental colitis. J. Mol. Med. 94, 545-556 (2016).

182. Muller, S. et al. Galectin-3 modulates T cell activity and is reduced in the inflamed intestinal epithelium in IBD. Inflamm. Bowel Dis. 12, 588-597 (2006).

183. Jensen-Jarolim, E. et al. The constitutive expression of galectin-3 is downregulated in the intestinal epithelia of Crohn's disease patients, and tumour necrosis factor alpha decreases the level of galectin-3-specific mRNA in HCT-8 cells. Eur. J. Gastroenterol. Hepatol. 14, 145-152 (2002).

184. Santucci, L. et al. Galectin-1 suppresses experimental colitis in mice. Gastroenterology 124, 1381-1394 (2003).

185. Hokama, A. et al. Induced reactivity of intestinal CD4(+) T cells with an epithelial cell lectin, galectin-4, contributes to exacerbation of intestinal inflammation. Immunity 20, 681-693 (2004).

186. Cummings, R. D. \& Pierce, J. M. The challenge and promise of glycomics. Chem. Biol. 21, 1-15 (2014).

187. Kearney C. J., et al. SUGAR-seq enables simultaneous detection of glycans, epitopes, and the transcriptome in single cells. Sci Adv 7 (2021).

188. Consortium, U. I. G. et al. Genome-wide association study of ulcerative colitis identifies three new susceptibility loci, including the HNF4A region. Nat. Genet. 41, 1330-1334 (2009).

189. Lauc, G. et al. Genomics meets glycomics-the first GWAS study of human $\mathrm{N}$-Glycome identifies HNF1alpha as a master regulator of plasma protein fucosylation. PLoS Genet. 6, e1001256 (2010).

190. de Lange, K. M. et al. Genome-wide association study implicates immune activation of multiple integrin genes in inflammatory bowel disease. Nat. Genet. 49, 256-261 (2017).

191. Anderson, C. A. et al. Meta-analysis identifies 29 additional ulcerative colitis risk loci, increasing the number of confirmed associations to 47. Nat. Genet. 43, 246-252 (2011).

192. Fonseca-Camarillo, G. \& Yamamoto-Furusho, J. K. Gene expression of solute carrier family 9 (sodium/hydrogen exchanger) 3, (SLC9A3) is downregulated in patients with ulcerative colitis. Inflamm. Bowel Dis. 18, 1197-1198 (2012).

193. Kruszewska, D. et al. Enteral crude red kidney bean (Phaseolus vulgaris) lectinphytohemagglutinin-induces maturational changes in the enterocyte membrane proteins of suckling rats. Biol. Neonate 84, 152-158 (2003).

194. Zhu, L. et al. and IL-10 receptor mutations in very early onset inflammatory bowel disease. Gastroenterol. Res. 10, 65-69 (2017).

195. Kotlarz, D. et al. Loss of interleukin-10 signaling and infantile inflammatory bowel disease: implications for diagnosis and therapy. Gastroenterology 143, 347-355 (2012).

196. Huang, H. et al. Fine-mapping inflammatory bowel disease loci to single-variant resolution. Nature 547, 173-178 (2017).

197. Graham, D. B. et al. TMEM258 Is a Component of the Oligosaccharyltransferase Complex Controlling ER Stress and Intestinal Inflammation. Cell Rep. 17, 2955-2965 (2016).

198. Liu, J. Z. et al. Association analyses identify 38 susceptibility loci for inflammatory bowel disease and highlight shared genetic risk across populations. Nat. Genet. 47, 979-986 (2015). 
199. Jostins, L. et al. Host-microbe interactions have shaped the genetic architecture of inflammatory bowel disease. Nature 491, 119-124 (2012).

200. Afzali, B. et al. BACH2 immunodeficiency illustrates an association between super-enhancers and haploinsufficiency. Nat. Immunol. 18, 813-823 (2017).

201. Duerr, R. H. et al. A genome-wide association study identifies IL23R as an inflammatory bowel disease gene. Science 314, 1461-1463 (2006).

202. Bouzid, D. et al. Polymorphisms in the IL2RA and IL2RB genes in inflammatory bowel disease risk. Genet Test. Mol. Biomark. 17, 833-839 (2013).

203. Mkhikian, $\mathrm{H}$. et al. Genetics and the environment converge to dysregulate $\mathrm{N}$-glycosylation in multiple sclerosis. Nat. Commun. 2, 334 (2011).

\section{ACKNOWLEDGEMENTS}

Research in the Parkos lab is supported by National Institute of Health $(\mathrm{NIH})$ grants R01DK072564 and R01DK079392. The authors would like to acknowledge Robin Kunkle for her help with figure illustration.

\section{AUTHOR CONTRIBUTIONS}

J.C.B. and C.A.P. contributed to the conception, writing and final approval of the manuscript.

\section{COMPETING INTERESTS}

The authors declare no competing interests.

\section{ADDITIONAL INFORMATION}

Correspondence and requests for materials should be addressed to Jennifer C. Brazil or Charles A. Parkos.

Reprints and permission information is available at http://www.nature.com/reprints

Publisher's note Springer Nature remains neutral with regard to jurisdictional claims in published maps and institutional affiliations. 